\title{
Cyclodextrins as Dominant Chiral Selective Agents in the Capillary Separation Techniques
}

\author{
Zoltán Juvancz ${ }^{*}$, Rita Bodáné-Kendrovics, Lajos Szente² ${ }^{2}$ Dóra Maklári³ \\ 1 Institute of Environmental Engineering, Óbuda University, H-1035 Budapest, 6 Doberdó Road, Hungary \\ 2 Cyclolab Ltd., H-1525 Budapest, P.O.B. 435, Hungary \\ ${ }^{3}$ Medical School Institute of Bioanalysis, University of Pécs, H-7624 Pécs, 1 Honvéd Street, Hungary \\ * Corresponding author, e-mail: juvancz.zoltan@rkk.uni-obuda.hu
}

Received: 18 February 2021, Accepted: 02 June 2021, Published online: 12 August 2021

\begin{abstract}
This review paper shows the dominant role of the cyclodextrins in the chiral separations using capillary columns (GC, SFC, CE). The cyclodextrins (CDs) have extremely broad chiral selectivity spectra because they have several different chiral recognition sites in various arrangements and various interaction modes. Their chiral selectivity features can further improve with their various substitutions. Their selectivities are moderate therefore they need high efficiency separations (capillary columns) for good chiral resolutions. The shape selectivity of cyclodextrins is also shown with non-chiral isomers too. The utility of the cyclodextrins is demonstrated with several examples based on the personal observations of authors and critical review of literature. The theoretical backgrounds of their chiral recognitions (e.g. H-bond interaction, inclusion, induced fit) are discussed in depth. This paper is not application oriented but is dealing with mostly on the physical and chemical background of separations using CDs.
\end{abstract}

Keywords

chiral separations, cyclodextrins, interaction types, chiral recognitions mechanisms, structure-selectivity relationships

\section{Introduction}

\subsection{Phenomenon of chirality}

The asymmetric molecules are called chiral molecules [1]. They are not superimposable with their mirror images. A chiral molecule and its mirror image molecule are enantiomers, or an enantiomeric pair.

Enantiomers have identical chemical and physical properties in an achiral (isotropic, amorphous) environment. However, the members of an enantiomeric pair have different properties in chiral (non-amorphous, anisotropic) environments (e.g., magnetic field, receptor sites and interactions with other asymmetric molecules). The molecules of living organisms (e.g., amino acids, sugars, receptors etc.) are dominantly asymmetric; therefore, the members of an enantiomeric pair can behave differently from each other in their biological effects.

The importance of introducing enantiopure drug substances, containing only one isomer has been recognized since the Contergan scandal [2]. Contergan (Thalidomide) medicine was a mixture of enantiomer molecules, in which one isomer produced a desirable antiemetic effect, whereas the other isomer of enantiomeric pair was toxic and produced teratogenicity side-effects [3]. From 1956 to 1962, more than 10,000 children were born with severe deformations (phocomelia) all over the world when their mothers had taken thalidomide during their pregnancy. Enantiomeric mixture of Robitussin, an over-the-counter cough medicine also caused lethal cases. Its useful isomer, dextromethorphan worked well, but its enantiomer, levomethorphan caused a lethal overdose effect because it has deposited in the patient's organs [4]. These cases and several other disturbances of applications of enantiomeric mixtures of pharmaceuticals forced the regulatory authority to approve only enantiopure drug substances [5]. It means a chiral drug must contain less than $0.1 \%$ of its optical isomer in the pharmaceutical products. Recently, the commercialization of enantiomer pure new pharmaceutical products is one of the fundamental regulatory requirements of the drug administration. The requirement of enantiomer pure agrochemicals is also adopted for some compounds. Frequently, only one enantiomer is responsible for the requested effects, but every isomer raises risk on the environment [6-8]. 


\subsection{Chiral selective separations}

The production and purity control of enantiomer pure products make their chiral selective analyses necessary. The chromatographic determination of the optical purity of a product requires the separation of the enantiomeric pair from each other. The separation of an enantiomer pair (chiral or optical separation) is generally based on a 3-point interaction model in chromatography [9-11]. According to the 3-point interaction model, one member of an enantiomeric pair (selectand) temporarily and simultaneously interacts with three groups of the chiral selector. The interacting groups of selector and selectand match exactly each other with their chemical nature and spatial arrangement in three points. The other member of this enantiomeric pair can interact only in 2-points with the selector. These differences of their interactions come from their non-uniform steric arrangement. The 3-point interaction is stronger than the 2-point interaction. The less strongly interacting enantiomer elutes first from columns having chiral selective stationary phases. An enantiomer separation needs tailor made interactions between the selector and selectand. No universal selector exists for chiral separations. Various enantiomeric pairs can be separated only by the application of such selectors which match well to the selectand both in steric formation and chemical characters. To find the appropriate selectors is very often the result of a trial-and-error process.

Generally, the chiral recognitions need certain rigidity of interacting molecules. The rather rigid chiral separation agents can separate narrow spectra of analytes, but they produce high selectivity values (e.g., amino acids). On the other hand, the flexible selectors can separate broad spectra of analytes but with low selectivity values.

One of the most effective chiral selective agents are the cyclodextrins (CDs) in chromatography. This article highlights the applications of CDs especially in the techniques using capillary columns [12-17]. CDs are also frequently applied in the other branches of analytical chemistry: sensors, fluorescence enhancement agents, absorbers in sample collections procedures, purification agents in sample pretreatment and as separation agents in HPLC. They are also used as solubilizers, control-release agents in pharmaceutical administrations, stereo specific catalysts in synthetic chemistry and remediation agents in environmental protection projects, but these topics are out of our scope $[18,19]$.

\section{Physical and chemical characterization of cyclodextrins}

The CDs are cyclic oligosaccharide molecule built up from alpha-D-glucopyranose units [19]. The glucose units linked together with $\alpha$-1,4-glycosidic linkages. The most important members of CDs consist of six, seven or eight D (+)-glucopyranose units, to which the letters Greek, $\alpha, \beta$ and $\gamma$ were assigned, respectively. The idealized structures of $\beta-\mathrm{CD}$ are in Fig. 1. The CDs have truncated cone shapes (Fig. $1 \mathrm{~A}$ ). The primary hydroxyl groups are located in the $\mathrm{C}(6)$ positions (Fig. 1 B), and they are around their narrower, upper rims (Fig. 1 A). The secondary hydroxyl groups are located in the $\mathrm{C}(2)$ and $\mathrm{C}(3)$ positions of glucose units

(Fig. 1 B) and they are on the wider rim (Fig. 1 A). There are continuous H-bond interactions among the secondary hydroxyl groups along the wider rim that give a semi rigid structure of the native CDs.

The sizes of cavities and molecular masses of cyclodextrins depend on the number of glucose units according to Table 1.

The CDs are capable of entrapping certain (called guest substances) in their nanosized cavities in non-covalent manner [19]. A tight fitting of the guest molecule in the cavity of CD results in a stabile inclusion complex. This is the reason of their use as molecular capsules in controlled release applications and formulations with protected guest against oxidation. A deeply and tightly immersed guest molecule

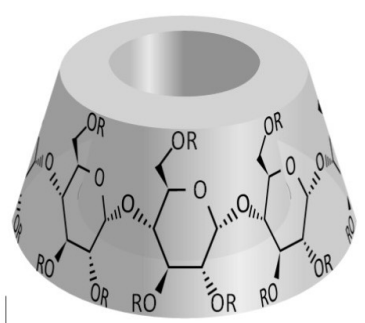

A

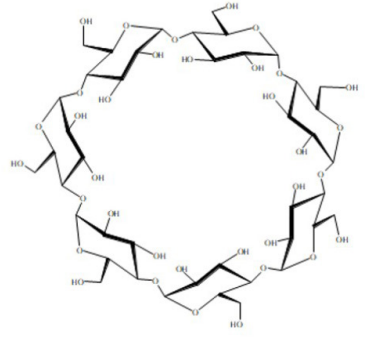

B
Fig. 1 The idealized structure of $\beta$-CD. A: geometrical arrangement (shape) of the molecule; B: chemical structure of molecule.

Table 1 The main physical characteristics of cyclodextrin molecules

\begin{tabular}{lcccc}
\hline \multirow{2}{*}{ Name } & \multirow{2}{*}{$\begin{array}{c}\text { Number of } \\
\text { glucopyra- } \\
\text { noses }\end{array}$} & \multicolumn{2}{c}{ Cavity (nm) } & Molecular \\
\cline { 3 - 4 } & 6 & 0.57 & 0.78 & 972 \\
\hline$\alpha$ & 7 & 0.78 & 0.78 & 1135 \\
$\beta$ & 8 & 0.95 & 0.78 & 1297 \\
$\gamma$ & & & & \\
\hline
\end{tabular}


creates a more stable complex than other guest molecules which are immersed to a lesser extent and loosely fitted in the cavity of a CD. Increasing the cavity size of CDs can tightly accommodate bigger molecules.

The cavity of CDs is a local a hydrophobic environment, less polar than water. The rims and outer surfaces of CDs show hydrophilic characters, offering H-bond, electron donor-acceptor interactions and enabling solubility of CDs in water. An apolar molecule becomes soluble in water, when it is included in the cavity of a CD [19].

The hydroxyl groups of native CDs can be chemically modified introducing various functional groups (e.g. alkyl, hydroxypropyl, acyl, carboxymethyl, phosphate, sulfate, amine and hydroxylamine etc.).

The $\mathrm{OH}$ group substitutions of CDs with functional groups result in changes in their sizes, shapes of their cavities and chemical characters [19]. These changes also cause shifting of their complexation properties comparing to their native compounds. Moreover, the substituents offer new interaction possibilities too. Introduction of an ionizable group (e.g., sulfobutyl, carboxylmethyl, amino) improves their water solubility and provides ionic features.

The substitutions of hydroxyl groups break the continuous H-bond structure of the secondary hydroxyls on the lower rim, which produces more flexible structures of the derivatized CDs.

Generally, statistical substitutions of CDs produce a large number of molecules, which differ from each other in number and positions of substituents. The random methylation of $\beta$-CD can produce more than 200,000 compounds differing from each other in their substitution rates and patterns [20]. Only the ten times methyl substituted $\beta$-CD can produce more than 88,000 isomers. Every member of such CD derivatives mixtures shows also alterations in their selectivity characters. The variety of molecules increases further when more types of chemical groups are among the substituents (e.g., terc-butyldimethylsilyl and acetyl, or amino and methyl). Such statistically derivatized CDs have reduced batch to batch reproducibility. An alternative synthesis produces a single isomeric derivative where the product has only one fixed structure $[21,22]$. These single isomeric $\mathrm{CD}$ derivatives show well-defined characteristics with excellent batch to batch reproducibility.

The native CDs are solid molecules. They decompose at higher temperatures (caramelization) without melting. Several derivatives of CDs, however, can melt above certain temperatures (e.g., permethyl $\beta$-CD [23], hexakis(2,3,6-tri-O-pentyl)- $\alpha$-cyclodextrin, octakis-(2,6-di-O-pentyl-3-O-butyryl)- $\gamma$-cyclodextrin) serving as good chiral stationary phases for GC [24]. It is also possible to have a CDs containing liquid phase when the $\mathrm{CD}$ is dissolved in a liquid matrix. Several CD derivatives (e.g., permethyl $\beta$-CD, octakis-(2,3-di-O-acetyl-6-O-tertierbutyl-dimethylsilyl)- $\gamma$-cyclodextrin) can be dissolved well in silicone polymer matrices and can be used as gas chromatographic (GC) stationary phases [16]. They also can be dissolved in water based or organic buffers for application of capillary electrophoresis (CE) [25].

\section{Isomer selectivity of cyclodextrins}

One of the most useful features of CDs is their shape selectivity from the perspective of the analytical chemistry [14-19, 24-32]. They can discriminate isomeric molecules. The steric arrangements of substitution groups of the guest compounds influence the strengths of interactions between the guest molecules and CDs.

\subsection{Selectivity of CDs toward the ring substitution isomers}

CDs can separate well the xylene ring substitution isomers, coming from their different inclusion properties [27, 29, 30]. The CDs can entrap the xylene isomers to different extents, producing different stability complexes with the included isomers. CDs can create stable inclusion complexes only with those molecules which fit well (immerse deeply) to the cavity of CDs. For example, only the para isomer of disubstituted benzene isomers (e.g., xylenes) can fit well to the cavity of $\alpha$-CD. The meta- and ortho- isomers are too wide to immerse deeply into the cavity of an $\alpha$-CD (Fig. 2).

It means that the $\alpha-C D$ forms the most stable complex with para isomers (longest retention time) and less stable complexes (shorter retention time) with ortho- and meta- isomers using an $\alpha$-CD containing stationary phase. Alternatively, the $\gamma$-CD can form the strongest complexes with ortho isomers of xylene. This is the reason why the permethylated $\alpha-\mathrm{CD}$ and $\beta$-CD containing stationary phases are excellent gas chromatographic phases for the separation of BTEX compounds (Fig. 3). In the field of environmental chemical analysis, the determination of
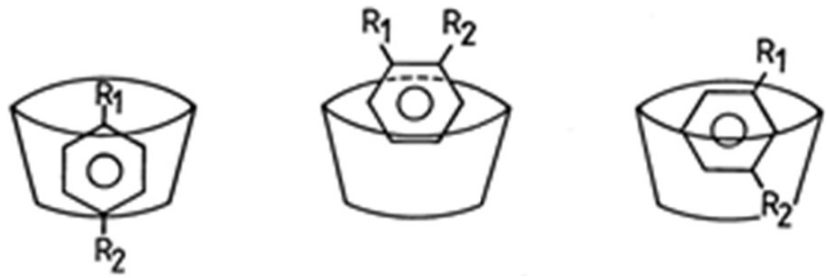

Fig. 2 The inclusion complexes of disubstituted benzene isomers and $\alpha$-CD. The most deeply immersed isomer (para) gives the most stable inclusion complex. 
carcinogenic benzene, toluene, various xylene isomers and ethylbenzene (BTEX) aromatic compounds has a high importance. The simultaneous separations of enantiomers and ring substitution isomers have also been demonstrated with chiral separation of psycho-active [27] and beta blocker metoprolol [30] drugs and their ring isomeric side products.

\subsection{Chiral selectivity of CDs}

The most important features of the CDs are their excellent chiral recognition characters from the standpoint of analytical chemistry [12-17, 19, 24-28, 30-35].

The extreme broad chiral selectivity spectra of the CD molecules make them the most popular chiral selectivity agents using capillary columns in GC [14, 16, 24, 31-34], CE [11, 13,15, 17, 22, 25, 26-28, 35], and SFC [36]. CDs can separate enantiomers with any functional groups. Even the enantiomers of saturated branched aliphatic hydrocarbons have been separated on a CD based stationary phase in GC $[24,37,38]$. There are no strict requirements for the structure of analytes for a successful chiral separation with CD-based chiral selectors [12]. The broad chiral recognition characters of CDs are based on the following phenomena:

- The CDs have numerous chiral centers - five in every glucose unit. The twisted forms of CDs cause non uniform shapes of glucose units as is demonstrated in Fig. 4. Functional groups of chiral centers have

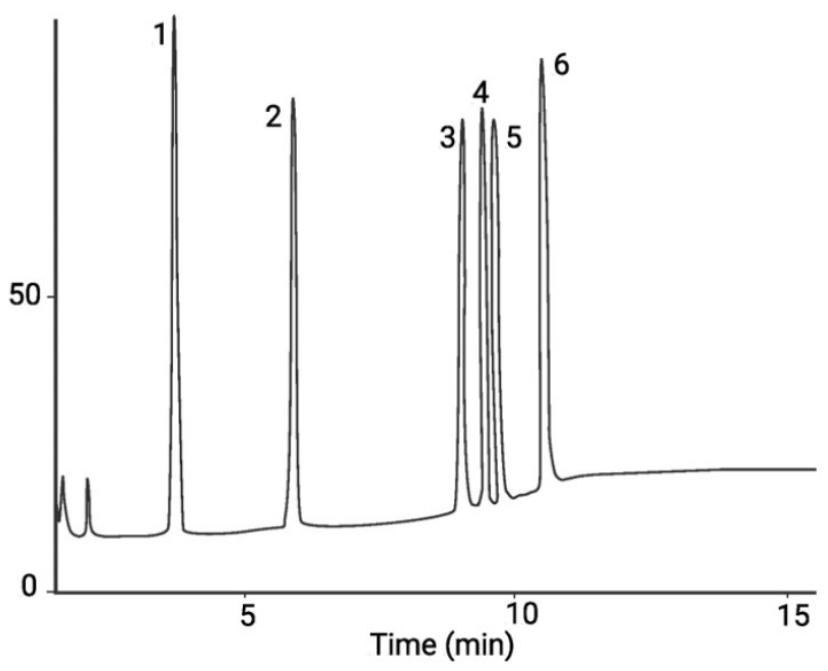

Fig. 3 GC Separation of BTEX compounds, Symbols: 1, benzene; 2, toluene; 3, o-xylene; 4, m-xylene; 5, ethylbenzene; 6 , p-xylene. Conditions: column, $25 \mathrm{~m} \times 0.22 \mathrm{~mm}$; stationary phase, Cydex-B $(0.25 \mu \mathrm{m}$, mixtures of silicone polymer and permethylated $\beta$-cyclodextrin); carrier, He (30 cm/sec); split ratio, 100:1; temperature program, $55^{\circ} \mathrm{C}(2 \mathrm{~min}) \rightarrow\left(2{ }^{\circ} \mathrm{C} / \mathrm{min}\right) \rightarrow 100{ }^{\circ} \mathrm{C}(5 \mathrm{~min})$. different orientations, and the chiral atoms have different distances from neighboring atoms in every glucose unit. Therefore, every glucose unit has 5 different chiral centers. Moreover, the shapes of the glucose units do not occur in same form from unit to unit. A $\beta$-CD has 35 different chiral recognition sites. It has been demonstrated that the cyclic CDs have broader selectivity spectra in GC than the linear amylose compounds which have a similar length and orientation of substituents in every glucose unit [39].

- The functional groups of substituents (phosphate, sulfate, amino, naphthyl, acetate) add a further interaction ability to the interaction potentials of native CDs $[17,25,28,30,35,40]$. The ionizable substituents (carboxy methyl, sulphobutyl, amino) make the ionic interactions possible. The aromatic substitutions offer П-П interactions for the chiral recognitions. Some substituent groups (such as hydroxypropyl and naphthyl-ethylcarbamoyl) add more chiral centers to CDs, further broadening their recognition spectra [19].

- As was mentioned, most CD derivatives are randomly substituted molecules. They are not uniform products but consist of a large number of isomers [20]. They differ from each other in their numbers and position of the substituents. Consequently, every isomer has different chiral recognition abilities. The substitution patterns of CDs can significantly influence the selectivity of the selectors [41-43]. The numbers and positions of methyl substitutions have significant impact on the chiral selectivity of the CD selectors [41]. Even migration reversals of separated enantiomers have been reported according to the degree of substitution of sulfated $\beta$-CD in CE [42].

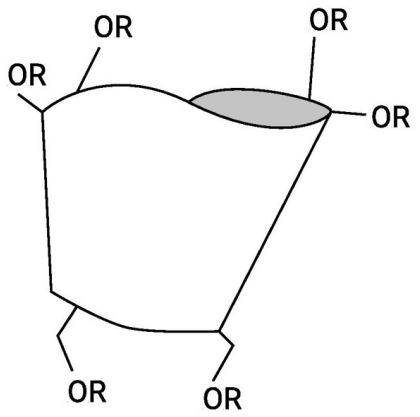

Fig. 4 The real shape of the derivatives cyclodextrins. It is a twisted truncated cone form with various bond lengths and directions. The R stands for various substituents. 
- The derivatized CDs have a rather flexible structure. CDs can change their shape to interact intimately (more strongly) with analytes with the so-called "induced fit" mechanism [19, 44]. The "induced fit" interactions further improve the chiral selectivity spectra of CDs. The flexible structures of CDs allow a recoil of the bulky O-tert-butyldimethyl silyl groups to cavity of CD if these substituents are in their 6 (primary) positions. The recoiled and normal arrangements of these structures result in significantly different shapes and chiral recognition properties of CDs [45].

- The ionizable CDs can change their selectivity spectra in accordance with their ionization states, as was observed in the case of phosphated $\gamma$-CD [46]. The phosphate substituted $\gamma$-CD almost loses its chiral selectivity toward to disopyramide in higher $\mathrm{pH}$ ranges $(\geq 4)$, since phosphated $\gamma$-CD becomes more ionized. The phosphated $\gamma$-CD shows higher chiral selectivity in the case of disopyramide in a less ionized state than when it is multiple ionized state. If the ionized state of $C D$ changes, the migration direction of the $\mathrm{CD}$ and its complex can also change their migration speed and directions in $\mathrm{CE}$. Such a change can result in reversal of migration of enantiomers [47].

- The types of mobile phases or background buffers influence the selectivity features of CDs. CDs change chiral recognition features, according to the separation media. They show three different types of chiral recognition features in accordance to the types of mobile phases: normal, reverse and polar organic [48]. In water-based media the $\mathrm{CD}$ can include simultaneously not only one host molecules, but a water molecule, too [19]. The included water modifies the chiral recognitions of CDs when compared to" dry"conditions. The role of the water has been observed among GC conditions too [49]. Chiral selectivity increased significantly when water vapor was added to the carrier gas in the case of methyl 2-chloropropanoate.

- The CDs can also separate enantiomers, having planar, or axial chirality not only enantiomers having central chirality [24].

Generally, the CDs favor the alpha positions of interacting groups of analytes from an asymmetric center. Such positions have increased rigidity of analyte around the asymmetric center than in the case of beta position [24]. CDs can also separate analytes having interacting groups at beta, gamma, and other positions. They can separate enantiomers of $\alpha, \beta, \gamma$ amino acids and hydroxyl acids, originated from their flexible structures [24]. The flexible structures of CDs, however, offer only moderate chiral selectivity. The large chiral selectivity needs significant rigidity of selectands as well as selectors. The $\alpha$-amino acid based chiral selectors (e.g., ChirasilVal) produce higher selectivity values towards the $\alpha$-amino acid enantiomers than the CDs, but they cannot separate B-amino acid enantiomers.

The great variety of chiral centers and induced fit can produce multimodal characteristics for the CDs. Many chiral recognition abilities of CDs make it difficult to predict the outcome chiral a resolution for a certain enantiomer pair. The multimodal character can offer more than one mode of interaction with an enantiomer pair [19, 50]. The $\gamma$-lactons can create complexes with CD in two ways. The cavity of $\mathrm{CD}$ can include the alkyl chain or the ring of a lactone. The inclusion of alkyl chain becomes dominant as the length of alkyl chains increase in the homolog series of $\gamma$-lactons. Therefore, elution reversal of enantiomers can be observed along the homologs of $\gamma$-lactons in GC [50].

\subsection{Key interactions of chiral recognition mechanisms of CDs}

Even the very weak London type dispersion interaction forces can play an important role in the chiral recognition process of CDs. This is the reason why CDs are appropriate for the chiral separations of enantiomers of saturated branched aliphatic hydrocarbons [24, 37, 38].

Several times the H-bonds interactions are responsible for chiral recognitions. In general, the enantiomers have H-donor properties (e.g., acids, amines, alcohols) show higher chiral selectivity than their derivatives (e.g., ester, acyl, and amide) [24, 28, 31]. The lone electron pairs of oxygen atoms of sugar units can create $\mathrm{H}$-bond interactions with the hydrogen donor groups of analytes. H-acceptor properties of sample molecules are also important in the chiral recognitions of the CDs [24, 25].

The aromatic ring system also helps in the chiral recognition process [19, 24, 31]. The aromatic parts of the analyzed enantiomers can fit the cavity of CDs. The inclusion strengths depend on the size of aromatic groups of sample and type of the CD [19]. In general, the $\alpha$-CDs can interact well with single aromatic ring. The naphthyl moieties interact better with $\beta$-CDs rather than other size CDs.

The dansyl derivatives of phenyl alanine show a 
protruding enantiomeric resolution (Rs:15.3) with permethylated amino $\beta$-CD, because the naphthyl containing substituent has rigid structure and good inclusion ability as was demonstrated in the CE practice [51].

The chiral recognition of CDs can be improved with achiral derivatives of the analyzed enantiomers. Such reactions create rigid derivatives of analytes to compensate for the moderate selectivity of the flexible structure of CDs [52]. The good chiral selectivity needs some rigidity for chiral discrimination as was already mentioned [10]. The separation of chiral vicinal diols can be improved by synthetizing their rigid cyclic carbonate derivatives with phosgene in GC $[52,53]$. Using phosgene, good chiral separations can be also achieved for chiral hydroxyl acids as 1,3-dioxolane (e.g., mandelic acid) and amino alcohols as oxazolidine (e.g., alprenolol), and diols as carbonate (e.g., guafenesine) cyclic derivatives. These cyclic derivatives are rather rigid and fit well to the cavity of permethylated $\beta$-CD.

The inclusion phenomenon is one of the key interactions of chiral recognition [19, 24, 28, 40, 45, 49, 51, 54-57] on several occasions, but on other occasions the inclusions do not play a role in chiral recognition $[24,28,40,54,58]$.

The water can play a significant role in these chiral selective inclusions as co-included molecules with analytes $[19,49]$.

Gas-chromatographic evidence also shows the chiral separations without inclusion phenomenon even in the case of functionless hydrocarbon. The crosslinking of the Chirasil-Dex GC stationary phase is a heat treatment [59]. Sometimes the heat treatments were too harsh, and the CD became pyrolyzed slightly. In these cases, the inclusion properties of permethylated $\beta$-CD diminished (Table 2).

The original Chirasil-Dex shows strong inclusion properties with c.-pinane. The retention of c.-pinane enantiomers (well penetrated molecules) are almost double that of undecane (non-penetrated) in spite of the two compounds having similar volatility. The "pyrolyzed" Chirasil-Dex loses its inclusion ability showing almost the same retention times for c.-pinane and undecane. However, the normal and "pyrolyzed" Chirasil-Dex columns produce similar chiral selectivity, showing that the chiral recognition

Table 2 Selectivity values c.-pinane/ undecane and +/- c.-pinane on normal and pyrolyzed Chirasil-Dex

\begin{tabular}{lcc}
\hline Column & $\begin{array}{c}\text { Selectivity } \\
\text { c.-pinane/undecane }\end{array}$ & $\begin{array}{c}\text { Selectivity }+/- \\
\text { c.pinane }\end{array}$ \\
\hline Chirasil-Dex & 1.890 & 1.093 \\
Pyrolyzed Chirasil-Dex & 1.008 & 1.080 \\
\hline
\end{tabular}

Column, $15 \mathrm{~m} \times 0.1 \mathrm{~mm}$; coated, Chirasil-Dex; temp., $80^{\circ} \mathrm{C}$ does not connect to the inclusion phenomenon in this case.

Several NMR [11, 17, 19, 24, 25, 28, 40, 55-58, 60, 61] studies were made to understand the chiral recognition and separation features among various cyclodextrin selectors and analytes. These NMR studies can show the interacting points and arrangements of participant of inclusion complexes. The NMR studies can refer, whether the analyte molecules are included in the cavity of $\mathrm{CD}$ or they interact with the outer surface of the CD [58, 61]. These studies also show that the different CDs, can include other parts of the analytes [55]. Conclusions from NMR experiments offer reasonable predictions for the separation success of other enantiomers. However, the observed differences of NMR data are not connected with the feasibility of chiral separations in a significant number of cases.

Molecular modeling, docking simulation studies were done for chiral recognition of CDs $[28,45,55,56,62]$ allowing deeper understanding of the chiral separation mechanisms. The flexible structures of CDs offer numerous conformations of the CDs. These conformers can create several complexes with the analytes having very similar stability energies. It is difficult to predict which complexes will be realized and which will show chiral recognition properties [45].

\subsection{Reversal of elution or migration orders of enantiomers using cyclodextrin selectors}

The first migration of minor enantiomer is important in the analysis of trace enantiomer impurities. If the minor enantiomer elutes first, the resolution 1.5 value is sufficient for exact determination of trace amounts of enantiomer impurities $(\sim 0.1 \%)$. On the other hand, when such a small enantiomer impurity elutes secondly, a higher resolution value $(>2.5)$ is required for the exact determination of such a low percentage of impurities. A slight tailing of the major peak can hide or coelute with the minor peak [63].

The amino acid-based selectors change the migration orders of the analyzed enantiomers using D-amino acids instead of L-amino acids in the selectors. No mirror image isomers of the CDs exist; therefore, elution reversal of enantiomers of the analytes cannot be managed by changing the CDs' chirality. There are several examples and tricks for elution or migration reversal of enantiomer pairs using CD selectors. These reversals can be managed more frequently with CDs than other selectors [47, 64-68].

The elution reversal can be achieved with changing the derivative of analytes in GC [66]. Alcoholic enantiomers and their acetate derivatives show enantiomeric reversal of several enantiomeric pairs on Chirasil-Dex stationary 
phase. Changing the $\mathrm{CD}$ chiral selectors can also result in enantiomer reversal in GC practice [67].

There are several methods to change the migration order of enantiomers $[25,28,30,42,47-64,65,68-70]$ in CE. Some of them are the following changes: the types of the CD selectors [30, 46, 68, 69], substitution rate of the CDs [42], size of the CDs [60], speed and direction of the electroosmotic flow [47], ionization state of analytes or selectors $[47,70]$.

\subsection{The reasons of the dominancy of CDs in chiral separations using capillary columns}

As already mentioned, the CDs have a flexible structure, therefore they have only moderate selectivity, however they can separate of a wide range of chiral analytes. The very high efficiencies (up to millions of theoretical plates) of capillary column techniques can compensate the moderate chiral selectivity of CDs to gain sufficiently high-resolution values. The high efficiency of GC come from the long (10-100 m), and relatively narrow $(0.1-0.5 \mathrm{~mm})$ diameter columns, and low viscosity (fast mass transfer) of the mobile phase. The flexible structured (helical) silicone polymers of stationary phases offer low resistance of mass transfer (fast diffusion rates). The high percentages (50-70\%) of the silicone matrices keep the efficiency high, and can solve reasonable portions (25-50\%) of the CDs selectors producing acceptable chiral selectivity.

The CE technique has a high efficiency, more than a million theoretical plates in the generally used (20$60 \mathrm{~cm}$ ) columns. The CD selectors are dissolved in the background buffer in CE techniques. The dispersed CD chiral selectors do not show any resistance of mass transfer. The analyte molecules transfer directly from selector molecules (pseudo stationary phase) into the background electrolyte (pseudo mobile phase) resulting in very fast transfer between the selector and background buffer. The analyte molecules do not need to diffuse through any stationary phase for mass transfer into "mobile phase". The dispersed states of selector molecules offer very short, less than $10 \mathrm{~nm}$ distances among the selector molecules, therefore the resistance of mass transfer is very low in the "mobile phase". These are the main reasons why the CE offers a very high efficiency in chiral separations. Such high efficiency allows the use of chiral selectors with moderate selectivity. The highly efficient columns make possible the resolution of enantiomeric pairs having only 0.1 $\mathrm{kJ} / \mathrm{M}(\alpha<1.01)$ interaction energy difference between the members of enantiomeric pairs in their interactions with the separation agents. The overwhelming parts of chiral separations use $\mathrm{CD}$ based selectors in $\mathrm{CE}$, coming from the wide separation spectra of CDs. Moreover, higher apparent selectivity $\left(\alpha_{\text {app }}\right)$ can be achieved than their thermodynamic selectivity with the manipulation with electroosmotic flow with oppositely charged selectors and analytes. Even infinite resolution can be managed between the members of enantiomeric pairs with the fine tuning of parameters [42].

The most popular polysaccharide polymeric chiral selectors of HPLC [71] are not appropriate for GC and CE applications. The HPLC practice uses layers of polysaccharide solid state molecules without any dilution showing high chiral selectivity values. They have high selectivity but show low efficiency. These rigid, dense polysaccharide macromolecule layers produce a high resistance of mass transfer in the stationary phase. They allow only a rather slow diffusion rate of analytes in the stationary phase. These slow mass transfers in stationary phases harmonize with the high resistance of mass transfer of the dense mobile phases and slow flow rates of mobile phases in HPLCs. These features of polysaccharide chiral HPLC, and short columns offer only moderate efficiency. The moderate selectivity of CDs requires highly efficient columns; therefore, CDs are less popular in HPLC practice. The polysaccharide stationary phases do not suit well the requirements of $\mathrm{GC}$ and $\mathrm{CE}$. The polysaccharide molecules have low solubility in silicone matrices (GC) and water-based background buffers (CE). The low solubility of polysaccharide molecules (low concentration) allows only moderate selectivity in GC and CE practice.

The CDs chiral selective stationary phases have limited applications in HPLC practice because they cannot be used in undiluted form in multilayer arrangements which result in moderate selectivity values. Their selectivity is rather low because they can cover the silica surface to a limited extent. Their large steric requirements do not allow for a dense coverage on a silica surface. Of course, there are several $\mathrm{CD}$ containing chiral stationary phases in the HPLC too [19, 72].

CDs are frequently used chiral selectors in mobile phase in HPLC practices $[18,19]$. However, the majority of chiral HPLC separations use chiral stationary phases instead of chiral mobile phase additives.

The overwhelming part of chiral SFC separations also used polysaccharide stationary phases in packed columns. The chiral capillary column SFC separations generally apply $\mathrm{CD}$ based stationary phases $[36,59]$. The open 
tubular columns SFC technique however is rarely used recently due to instrumental difficulties of this method.

\section{Application of cyclodextrins in chiral separations 4.1 Gas chromatographic applications of cyclodextrins} The high efficiency values of capillary GC columns require only limited chiral selectivity for baseline resolutions. Even enantiomer pairs having only $1.01 \alpha$ value were separated on $\mathrm{CD}$ containing columns $[31,32]$. The $\alpha$ values remain under 1.2 on most cases using CD containing columns. Only in one case $\alpha$ was measured with a value of 10.6 when a CD based stationary phase was used [73].

The measured $\alpha$ (corrected retention time ratio) values of enantiomers do not show the real interaction energy differences among the member of enantiomer pairs, using stationary phases of mixtures of silicone polymers and CDs [73, 74]. The retention times of enantiomer compose two factors: retentions in silicon matrix (non-chiral interactions), and retentions in CDs (chiral interactions). The measured retentions of the enantiomer pairs have to be corrected with the non-chiral retention to get real base for the calculations of chiral selection powers.

The chiral separations were done mostly in enthalpic region, which means the chiral separations increase exponentially with decreasing temperature [24, 34, 74]. Chiral separation is extremely rare in an entropic region (above Tiso) using CD based stationary phase [75]. Nonlinear Van't Hoff plot was measured (ln $\alpha$ in the function of $1 / \mathrm{T}$ ) for enantiomers of trifluoroacetate ethyl ester derivatives of some $\alpha$-amino acids on Chirasil-Dex [75]. Probably more than one chiral selective interaction played a role on these occasions which have different temperature dependencies.

More than $90 \%$ of GC chiral separations have been done on CD containing stationary phases [14, 16, 24, 31-33, $76,77]$. Only the amino acid based Chirasil-Val stationary phase is used in the everyday chiral selective GC practice, which is not $\mathrm{CD}$ based selector. The uses of Chirasil-Val are mostly limited to chiral analysis of $\alpha$-amino acids. The chiral separations of proteomic amino acids, however, can be carried out with CD based selectors, too [24]. The chiral selectivity values of $\mathrm{CD}$ containing stationary phases are moderate, therefore high separation power of long capillary columns are required for the appropriate resolutions. However, the selectivity of enantiomers can be improved with the achiral derivatization agents [31].

The broad selectivity spectra of CDs offer chiral separations for enantiomers having different structures, sizes, and functional groups. The CDs can separate every class of enantiomers, even the functionless hydrocarbons [24].

One of the outstanding properties of CDs is that they can separate unfunctionalized chiral alkane enantiomers $[24,37,38]$. This feature of the CDs makes it appropriate to find the origin of asymmetric molecules even on Mars and in meteors [38].

The used CDs are thermo-stable derivatives of native CDs in gas chromatography practices. They are applied in the following forms: molten states, dissolved in achiral matrices or chemically bonded to silicone polymers [31]. At the beginning, the molten derivatives of $\mathrm{CD}$ were used in the GC practice. Some of them are liquid even in room temperature [24], or they were used in their super cooled states under their melting point. These stationary phases, however, have moderate efficiency, because the resistance of mass transfer values are rather high in stationary phase of undiluted CD derivatives. Some partially derivatized CD have been introduced as chiral stationary phase, but their underivatized $\mathrm{OH}$ groups cause limited thermal stability and moderate batch-to-batch reproducibility [67, 76].

An up-to-date CD containing stationary phase consists of a chiral selective CD and a silicone polymer matrix [16, 24, 31-33, 44, 75, 76].

The silicone matrix offers a high efficiency above 5,000 theoretical plates/meter, and the CD gives appropriate chiral selectivity for the stationary phase as Fig. 5 presents.

Fig. 5 shows well the separations of the enantiomers as well as the separations of cis (first and fourth peaks), and trans (second and third peaks) positional isomers of chrysantemic acids. The stationary phase has an optimal rate between the polymer matrix and chiral selector. The $80 \%$ dimethyl phenyl moderately polar polysiloxane matrix offers a high efficiency and enough solubility for $20 \%$ permethylated $\beta$-cyclodextrin, which gives good chiral selectivity. The moderate polarity matrix allows the separation of relatively volatile acids without derivatization at moderate analysis temperatures.

The chiral selectivity values of stationary phases show level up characters in the function of the concentration of CDs [74]. The selectivity values of the stationary phases do not increase above certain concentrations of the CDs. When the concentration of the CD is too high in the stationary phase, probably, the chiral recognition sites of CD molecules interact with other CD molecules instead of analytes. Moreover, too high CD contents result in decreased efficiency. The silicon matrix is also good to solve solid $\mathrm{CD}$ derivatives in a liquid matrix as was 


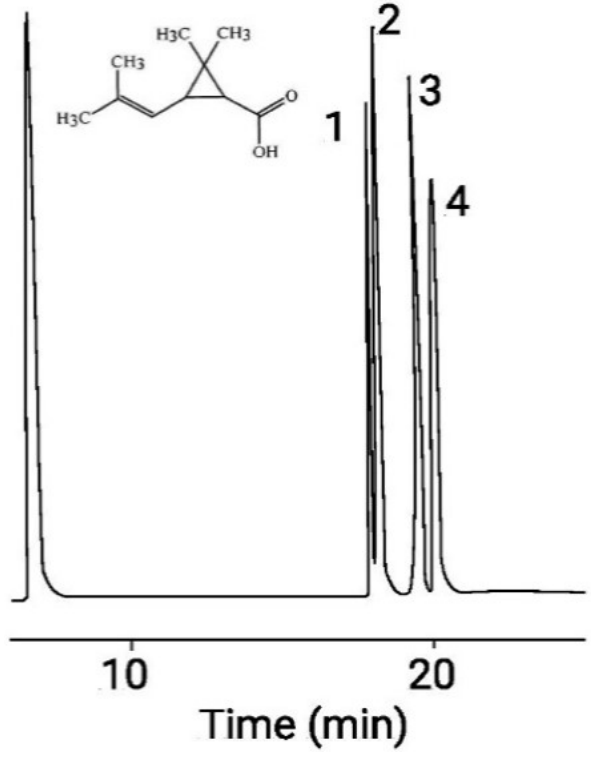

Fig. 5 Separation of chrysantemic acid isomers cis-trans and $R / S$ ) as free acids. Conditions: instrument, HP 5890/II; column, $25 \mathrm{~m} \times 0.22 \mathrm{~mm}$; stationary phase, Cydex-B $(0.25 \mu \mathrm{m})$; carrier, $\mathrm{H}_{2}(50 \mathrm{~cm} / \mathrm{sec})$; temp, $120^{\circ} \mathrm{C}$. Elution order: 1 , cis $(+) ; 2$, trans $(+) ; 3$, trans $(-) ; 4$, cis $(-)$.

experienced in the cases of terc-butyldimethylsilyl substituted CDs. Those CD selectors, which were used in a molten state originally, are recently applied mixtures of $50 \%$ $\mathrm{CD}$ and $50 \%$ silicone matrices [78].

The enantiomers can be analyzed at lower temperatures with mixtures of CDs and silicon stationary phases than the analysis temperature of molten CDs. The silicone matrices have a lower polarity than the CDs, allowing a lower analysis temperature. The low analysis temperatures are advantageous from the point of view of chiral selectivity in GC [16, 24, 25]. Namely the chiral selectivity values increase exponentially with the decreasing analyses temperature $[24,34,74]$.

The CD based chiral selectors can be chemically bonded to a silicone matrix too [52]. Immobilized Chirasil-Dex and other anchored chiral phases have advantages over the silicone polymer and chiral selector mixtures: they have a broader usable temperature range and show insolubility after cross-linking [59]. The cross-linked Chirasil-Dex can be used in SFC mode also. Anchoring the CDs to a silicon matrix needs a dedicated, tedious synthesis method. This is the reason why the mixture versions ( $\mathrm{CD}$ and silicon matrix) remain the most often used forms of the $\mathrm{CD}$ containing gas chromatographic stationary phases. Several tens of thousands of enantiomer pairs have been separated on CD based selectors in GC. There are no strict requirements for the arrangement of functional groups around the chiral centers of analytes using $\mathrm{CD}$ based selectors [24, 32].

The CD containing stationary phases have been used in narrow bore and microcolumn $\mathrm{GC}$ to improve the efficiency of the columns [34, 52, 79-81]. A $50 \mathrm{~cm}$ x $50 \mu \mathrm{m}$ column coated with immobilized Chirasil- $\gamma$-Dex was appropriate to separate enflurane enantiomers within 10 seconds [34]. A planar column $(168 \mathrm{~cm} \times 50 \mu \mathrm{m} \times 50 \mu \mathrm{m})$ was applied successfully to separate enantiomers of lavender and bergamot essential oils [79]. The narrow bore columns can speed up the analyses but result in only limited loadability of the columns.

Several hundred components using a two-dimensional gas chromatographic system can be separated during one analysis [82]. Such systems use mostly CD based chiral columns as a second dimension in the case of enantiomer separations.

\subsection{Application of cyclodextrins in capillary electrophoresis}

Basically, the CE is not a chromatographic method however, the chiral selective CE separations also require distribution processes. The different interaction energies of the members of an enantiomer pair with a selector result in different residence times in free and associated forms with selectors. When the free and associated forms do not have equal migration speeds, a chiral separation is possible [83]. Neutral selectors cannot separate neutral enantiomers because they have the same migration speed as electroosmotic flow in any form. Enantiomers can be separated by $\mathrm{CE}$ technique, when they have an equal binding constant with a CD selector in some cases $[17,84,85]$. On these occasions, the complexes of the enantiomers have different mobilities due to the different steric arrangements of the analytes in the CDs.

The low analysis temperature is one of the reasons why the $\mathrm{CE}$ is very advantageous in chiral analysis [13, 25]. It has to be highlighted that chiral selectivity of selectors increase exponentially with decreasing temperature [34].

Capillary electrophoresis (CE) is an ideal method for chiral analysis $[12,13,15,17-28,35,58-66,83-85]$. Generally, the chiral selectors are dissolved in a background buffer as a pseudo stationary phase offering high speed mass transfers. The CE has the following advantages in chiral analyses:

- high efficiency (> 1,000,000 plates),

- low analysis temperature,

- fast analysis,

- quick method developments, 
- low material consumption of analytes,

- low material consumption of buffer,

- flexible working parameters,

- simplified sample preparation from biological matrices,

- cheap operational expenses,

- partially filling method allows the use of selectors with high UV absorption features.

The very low material consumption allows using very expensive CD derivatives (e.g., sulfate or amine substituted CDs). A $10 \mathrm{ml}$ buffer is enough for one day's work with $0.5-15 \mathrm{mM}$ chiral selector concentrations.

The CDs are the most frequently used chiral selectors in CE $[12,13,15,17,25,26,28,35,58,83-85]$. According to the literature, more than two thirds of chiral separations have been made with $\mathrm{CD}$ chiral selectors in capillary electrophoresis. The capillary electrophoresis uses native CDs as well as derivatives of CDs. More than 100 derivatized CDs have been used for chiral separations in the CE practices $[12,13,25,26,28]$. The very broad selectivity spectra of CDs, very high efficiency of CE measurements and low analysis temperatures are the reasons why the CD applications are very popular in the chiral selective analyses.

Recently the charged CDs become more and more popular $[13,15,21,25,64,84]$. Sometimes a $0.5 \mathrm{mM}$ ionized $\mathrm{CD}$ chiral selector is enough for baseline separation of oppositely charged enantiomers (Fig. 6).

This good selection was possible because the CD and the analytes have opposite charges. The analytes have opposite migration directions in free and temporarily

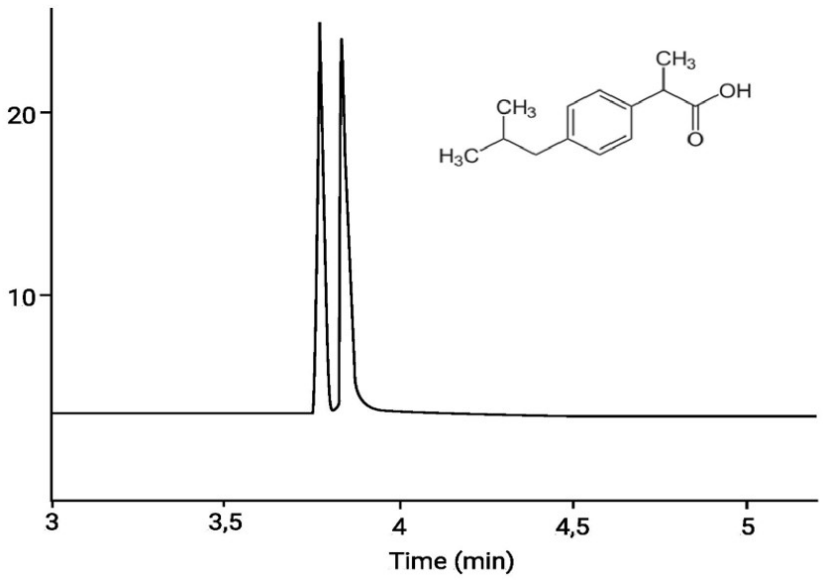

Fig. 6 Separation of enantiomers of ibuprofen using heptakis(2,3dimethyl-6-amino-6-deoxy)- $\beta$-cyclodextrin, Parameters: Column, $58.5 \mathrm{~cm}$ x $50 \mathrm{~mm}$ I.D. FSOT; detector, UV $214 \mathrm{~nm}$; background electrolyte, $\mathrm{pH} 5 ; 40 \mathrm{mM}$ boric, $40 \mathrm{mM}$ acetic and $40 \mathrm{mM}$ phosphoric acid buffers in ratio of 1:2:2; selector, $0.5 \mathrm{mM}$ heptakis(2,3-dimethyl-6amino-6-deoxy)- $\beta$-cyclodextrin; potential $-30 \mathrm{kV}$. diastereomeric complexed states causing pseudo elongation of separation length. This effect is also responsible for the higher chiral apparent selectivity values $\left(\alpha_{\text {app }}\right)$ rather than expected from thermodynamic data. $[13,15,25$, $42,57]$. Even infinite resolution has been reported using certain concentrations of an oppositely charged CD selector and analytes [42]. In this case, the strongly bonded enantiomer follows the migration direction of a charged selector which is opposite to its own migration direction of analyte. The weakly bond enantiomer follows its own migration direction because it spends less time in a complexed state than the strongly bonded enantiomer.

Moreover, the charged CD can separate neutral and charged enantiomers, but neutral CDs can separate only charged ones [25].

The overwhelming part of the $\mathrm{CD}$ derivatives are statistically substituted. For the sake of good reproducibility however, single isomeric derivatives CDs have also been introduced [38, 49, 68]. The statistically substituted CD derivatives have a broader selectivity spectrum [13]. Applying a dual CD system can further improve the chiral separations [86-89]. Some combinations show synergetic selectivity. A dual system can separate enantiomers with more than one chiral center. One of the CDs is selective for the first chiral center and the other $\mathrm{CD}$ is selective for another chiral center. There are some other synergetic effects which have also been reported using two CDs or combinations of a CD with other types of selectors. Some dual systems were appropriate to separate neutral enantiomers, which have the same interaction energy with charged CDs. Migration speed of the charged complexes offer a different migration speed other than the electroosmotic flow. In these cases, the complexes of a neutral enantiomer with neutral cyclodextrins show a difference in their stabilities, but they have the same migration speed with electroosmotic flow. The different residence times of the enantiomers in the neutral complex also cause shifting of the residence times of the analytes in the charged complexed states too.

The CE is also appropriate for the determination of yields of diastereomeric salt resolution during one analysis [90]. The fast electroosmotic flow produces migration in the direction of the cathode for the anions and cations, and simultaneously the chiral separations can also take place. Such determinations are possible only in capillary electrophoretic experiments (Fig. 7).

The counter-current, or partial filling method, offers unique ability to CE/MS. This method applies selectors with migration direction towards the injection point, so 


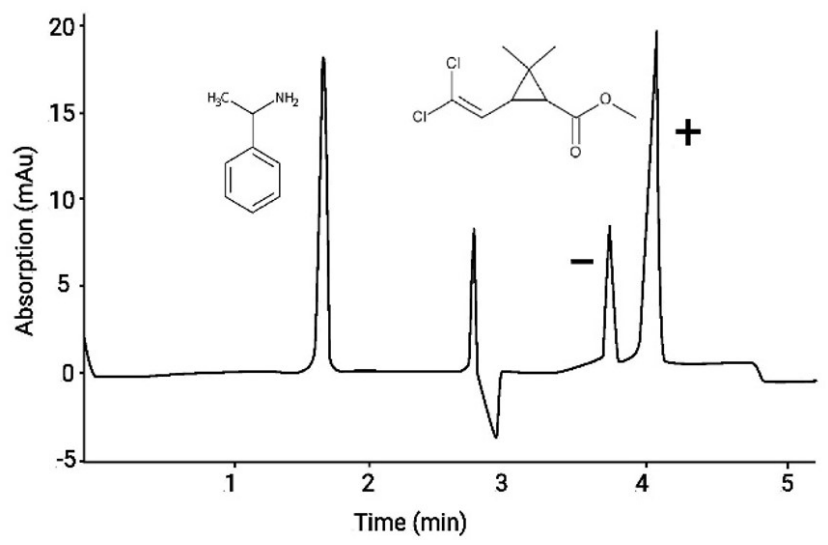

Fig. 7 Simultaneous determination of products of diastereomeric salt resolution of cis-permethrinic acid with (R)-1-phenylethylamine Conditions: $33.5 / 25 \mathrm{~cm} \times 50 \mu \mathrm{m}$, FSOT capillary; $+20 \mathrm{kV}$ applied voltage; $200 \mathrm{~nm}$ detection wavelength; temp. $25^{\circ} \mathrm{C}$; background electrolyte, $50 \mathrm{mM}$ Britton-Robinson buffer ( $\mathrm{pH} 7.2$ ); chiral selector, $10.0 \mathrm{mM}$ randomly methylated-(6)- monoamino-deoxy-(6)-cyclodextrin chiral selector, with permission from [90].

the selectors do not disturb UV detection or MS measurements [73].

The microfluidic chip technology is also used in capillary electrophoresis [91-94]. A chip CE instrument has been developed to explore bioorganic signatures from extraterrestrial samples [91, 93]. The microchip capillary electrophoresis allows for very fast analyses. For example, the dansylated amino acid enantiomers were separated within 1 second on such a device [94].

Chemically bonded CD containing stationary phases have also been applied as a unified approach including

\section{References}

[1] Poppe, L., Nógrádi, M., Nagy, J., Hornyánszky, G., Boros, Z. "Stereochemistry and Stereoselective Synthesis: An Introduction", Wiley, Weinheim, Germany, 2016.

[2] Kim, J. H., Scialli, A. R. "Thalidomide: The Tragedy of Birth Defects and the Effective Treatment of Disease", Toxicological Sciences, 122(1), pp. 1-6, 2011. https://doi.org/10.1093/toxsci/kfr088

[3] Teo, S. K, Colburn, W. A., Tracewell, W. G., Kook, K. A., Stirling, D. I., Jaworsky, M. S., Scheffler, M. A., Thomas, S. D., Laskin, O. L. "Clinical Pharmacokinetics of Thalidomide", Clinical Pharmacokinetics, 43(5), pp. 311-327, 2004. https://doi.org/10.2165/00003088-200443050-00004

[4] Rouhi, A. M. "Chirality at work", Chemical and Engineering News, 81(18), pp. 56-61, 2003.

[5] Food and Drug Administration, Department of Health and Human Services, USA "FDA's policy statement for the development of new stereoisomeric drugs", Chirality, 4(5), pp. 338-340, 1992. https://doi.org/10.1002/chir.530040513 electrochromatography $[34,80,81]$. The electrochromatography method has only limited use in chiral separations. One of the advantages of capillary electrophoresis is the very high efficiency value which is partly lost using electrochromatographic technique. The stationary phase shows significant resistance of mass transfer in electrochromatography which results in decreased efficiency comparing CE methods.

\section{Conclusion}

The cyclodextrins are versatile chiral selective agents. Their flexible structures offer very broad chiral recognition properties in the case of many enantiomers having different structures and functional groups. Various derivatives of cyclodextrins extend their application range further out. The flexible structures of cyclodextrins produce moderate chiral selectivity which can be compensated by the high efficiency of capillary columns of GC and CE to achieve good resolutions of the enantiomer pairs. This is the reason why the overwhelming part of the chiral separations uses cyclodextrins as chiral selective agents in GC and CE.

\section{Acknowledgements}

The authors thank the valuable help of András Szeder, and Róbert Iványi. The fruitful consultations with László Jicsinszky are acknowledged. The regardful advices of György Mikéta helped a lot in the editing processes The careful linguistics and grammar corrections of Graham Welsh are highly appreciated, they improved the quality of manuscript.

[6] Jeschke, P. "Current status of chirality in agrochemicals", Pest Management Science, 74(11), pp. 2389-2404, 2018. https://doi.org/10.1002/ps.5052

[7] Chen, S., Zhang, L., Chen, H., Chen, Z., Wen, Y. "Enantioselective Toxicity of Chiral Herbicide Metolachlor to Microcystis aeruginos", Journal of Agricultural and Food Chemistry, 67(6), pp. 1631-1637, 2019.

https://doi.org/10.1021/acs.jafc.8b04813

[8] Maia, A. S., Ribeiro, A. R., Castro, P. M. L., Tiritan, M. E. "Chiral Analysis of Pesticides and Drugs of Environmental Concern: Biodegradation and Enantiomeric Fraction", Symmetry, 9(9), Article number: 196, 2017. https://doi.org/10.3390/sym9090196

[9] Dalgliesh, C. E. "756. The optical resolution of aromatic amino-acids on paper", Journal of Chemical Society (resumed), pp. 3940-3942, 1952. https://doi.org/10.1039/jr9520003940 
[10] Meyer, V. R., Rais, M. "A vivid model of chiral recognition", Chirality, 1(2), pp. 167-169, 1989. https://doi.org/10.1002/chir.530010211

[11] Scriba, G. K. E. "Chiral recognition in separation science - an update", Journal of Chromatography A, 1467, pp. 56-78, 2016. https://doi.org/10.1016/j.chroma.2016.05.061

[12] Juvancz, Z., Szejtli, J. "The role of cyclodextrins in chiral selective chromatography", TrAC Trends in Analytical Chemistry, 21(5), pp. 379-388, 2002. https://doi.org/10.1016/S0165-9936(02)00506-X

[13] Juvancz, Z., Bodáné Kendrovics, R., Iványi, R., Szente, L. "The role of cyclodextrins in chiral capillary electrophoresis", Electrophoresis, 29(8), pp. 1701-1712, 2008. https://doi.org/10.1002/elps.200700657

[14] Menestrina, F., Ronco, N. R., Castells, C. B. "Enantioselective gas chromatography with functionalized cyclodextrins as chiral selectors. Fundamentals of the measurement of absolute association constants using capillary columns", Journal of Chromatography A, 1467, pp. 482-489, 2016. https://doi.org/10.1016/j.chroma.2016.06.071

[15] Saz, J. M., Marina, M. L. "Recent advances on the use of cyclodextrins in the chiral analysis of drugs by capillary electrophoresis", Journal of Chromatography A, 1467, pp. 79-94, 2016. https://doi.org/10.1016/j.chroma.2016.08.029

[16] V. Schurig "Gas Chromatography: Chiral Separations", In: Worsfold, P., Poole, C., Townshend, A., Miró, M. (eds.) Encyclopedia of Analytical Science, Elsevier, Amsterdam, Netherland, 2019, pp. 107-117. https://doi.org/10.1016/B978-0-12-409547-2.14501-9

[17] Fanali, S., Chankvetadze, B. "Some thoughts about enantioseparations in capillary electrophoresis", Electrophoresis, 40(18-19), pp. $2420-2437,2019$. https://doi.org/10.1002/elps.201900144

[18] Szente, L., Szemán, J. "Cyclodextrins in Analytical Chemistry: Host-Guest Type Molecular Recognition", Analytical Chemistry, 85(17), pp. 8024-8030, 2013. https://doi.org/10.1021/ac400639y

[19] Szejtli, J., Osa, T. "Comprehensive Supramolecular Chemistry, Volume 3: Cyclodextrins", Pergamon, London, UK, 1999.

[20] Wenz, G. "Cyclodextrins as Building Blocks for Supramolecular Structures and Functional Units", Angewandte Chemie International Edition, 33(8), pp. 803-822, 1994. https://doi.org/10.1002/anie.199408031

[21] Iványi, R., Jicsinszky, L., Juvancz, Z. "Chiral separation of pyrethroic acids with single isomer permethyl monoamino $\beta$-cyclodextrin selector", Electrophoresis, 22(15), pp. 3232-3236, 2001. https://doi.org/10.1002/1522-2683(200109)22:15<3232::AIDELPS3232>3.0.CO;2-G

[22] Fejős, I., Kalydi, E., Malanga, M., Benkovics, G., Béni, Sz. "Single isomer cyclodextrins as chiral selectors in capillary electrophoresis", Journal of Chromatography A, 1627, Article number: 461375, 2020. https://doi.org/10.1016/j.chroma.2020.461375

[23] Juvancz, Z., Alexander, G., Szejtli, J. "Permethylated $\beta$-cyclodextrin as stationary phase in capillary gas chromatography", Journal of High Resolution Chromatography, 10(2), pp. 105-107, 1987. https://doi.org/10.1002/jhrc.1240100214
[24] König, W. A. "Gas chromatographic enantiomer separation with modified cyclodextrins", Hüthig, Heidelberg, Germany, 1992.

[25] Chankvetadze, B. "Capillary electrophoresis in chiral analysis", Wiley, Chichester, UK, 1997.

[26] ̌ezanka, P., Navrátilová, K., Řezanka, M., Král, V., Sýkora, D. "Application of cyclodextrins in chiral capillary electrophoresis", Electrophoresis, 35(19), pp. 2701-2721, 2014. https://doi.org/10.1002/elps.201400145

[27] Schmid, M. G., Hägele, J. S. "Separation of enantiomers and positional isomers of novel psychoactive substances in solid samples by chromatographic and electrophoretic techniques - A selective review", Journal of Chromatography A, 1624, Article number: 461256, 2020. https://doi.org/10.1016/j.chroma.2020.461256

[28] Scriba, G. K. E. "Chiral recognition in separation sciences. Part I: Polysaccharide and cyclodextrin selectors", TrAC Trends in Analytical Chemistry, 120, Article number: 115639, 2019. https://doi.org/10.1016/j.trac.2019.115639

[29] Sybliska, D., Kościelski, T. " $\beta$-cyclodextrin as a selective agent for the separation of o-, $\mathrm{m}$ - and $\mathrm{p}$-xylene and ethylbenzene mixtures in gas-liquid chromatography", Journal of Chromatography A, 261, pp. 357-362, 1983. https://doi.org/10.1016/S0021-9673(01)87963-2

[30] Juvancz, Z., Jicsinszky, L., Markides, K. E. "Chiral analysis of metoprolol and its by-products by capillary electrophoresis", Journal of Microcolumn Separations, 11(10), pp. 716-722, 1999. https://doi.org/10.1002/(SICI)1520-667X(1999)11:10<716::AIDMCS4>3.0.CO;2-I

[31] Juvancz, Z., Petersson, P. "Enantioselective gas chromatography", Journal of Microcolumn Separations, 8(2), pp. 99-114, 1996. https://doi.org/10.1002/(SICI)1520-667X(1996)8:2\%3c99::AID$\mathrm{MCS} 3 \% 3 \mathrm{e} 3.0 . \mathrm{CO} ; 2-2$

[32] Schurig, V. "Use of derivatized cyclodextrins as chiral selectors for the separation of enantiomers by gas chromatography", Annales Pharmaceutiques Françaises, 68(2), pp. 82-98, 2010. https://doi.org/10.1016/j.pharma.2009.11.004

[33] Ribeiro, C., Gonçalves, R., Tiritan, M. E. "Separation of Enantiomers Using Gas Chromatography: Application in Forensic Toxicology, Food and Environmental Analysis", Critical Reviews in Analytical Chemistry, pp. 1-25, 2020. https://doi.org/10.1080/10408347.2020.1777522

[34] Schurig, V. "Separation of enantiomers by gas chromatography", Journal of Chromatography A., 906(1-2), pp. 275-299, 2001. https://doi.org/10.1016/S0021-9673(00)00505-7

[35] Yu, R. B., Quirino, J. P. "Chiral Selectors in Capillary Electrophoresis: Trends during 2017-2018", Molecules, 24(6), Article number: 1135, 2019. https://doi.org/10.3390/molecules24061135

[36] Juvancz, Z., Markides, K. E. "Enantiomer Separation Using Supercritical Fluid Chromatography", LC GC International, 5(4), pp. 44-56, 1992.

[37] Sicoli, G., Kreidler, D., Czesla, H., Hopf, H., Schurig, V. "Gas chromatographic enantioseparation of unfunctionalized chiral alkanes: A challenge in separation science (overview, state of the art, and perspectives)", Chirality, 21(1), pp. 183-198, 2009. https://doi.org/10.1002/chir.20638 
[38] Meierhenrich, U. J., Nguyen, M. J., Barbier, B., Brack, A., Thiemann, W. H. P. "Gas chromatographic separation of saturated aliphatic hydrocarbon enantiomers on permethylated $\beta$-cyclodextrin", Chirality, 15(S1), 2002, pp. S13-S16, 2003. https://doi.org/10.1002/chir.10269

[39] Schurig, V., Nowotny, H. P., Schleimer, M., Schmalzing, D. "Gas chromatographic enantiomer separation on per- $n$-pentylated amylose", Journal of High Resolution Chromatography, 12(8), pp. 549-551, 1989 . https://doi.org/10.1002/jhrc.1240120815

[40] Gogolashvili, A., Tatunashvili, E., Chankvetadze, L., Sohajda, T., Szeman, J., Gumustas, M., Ozkan, S. A., Salgado, A., Chankvetadze, B. "Separation of terbutaline enantiomers in capillary electrophoresis with cyclodextrin-type chiral selectors and investigation of structure of selector-selectand complexes", Journal of Chromatography A, 1571, pp. 231-239, 2018. https://doi.org/10.1016/j.chroma.2018.08.012

[41] Varga, E., Benkovics, G., Darcsi, A., Várnai, B., Sohajda, T., Malanga, M., Béni, S. "Comparative analysis of the full set of methylated $\beta$-cyclodextrins as chiral selectors in capillary electrophoresis", Electrophoresis, 40(21), pp. 2789-2798, 2019. https://doi.org/10.1002/elps.201900134

[42] Rudaz, S., Calleri, E., Geiser, L., Cherkaoui, S., Prat, J., Veuthey, J. L. "Infinite enantiomeric resolution of basic compounds using highly sulfated cyclodextrin as chiral selector in capillary electrophoresis", Electrophoresis, 24(15), pp. 2633-2641, 2003. https://doi.org/10.1002/elps.200305481

[43] Řezanka, M., Řezanka, P., Sýkora, D., Jindřich, J., Král, V. "Impact of substituent position in monosubstituted $\alpha$-cyclodextrins on enantioselectivity in capillary electrophoresis", Journal of Separation Science, 35(7), pp. 811-815, 2012. https://doi.org/10.1002/jssc.201101034

[44] Saenger, W., Noltemeyer, M., Manor, P. C., Hingerty, B., Klar, B. " "Induced-fit"-type complex formation of the model enzyme $\alpha$-cyclodextrin", Bioorganic Chemistry, 5(2), pp. 187-195, 1976. https://doi.org/10.1016/0045-2068(76)90007-9

[45] Kobor, F., Angermund, K., Schomburg, G. "Molecular modelling experiments on chiral recognition in GC with specially derivatized cyclodextrins as selectors", Journal of High Resolution Chromatography, 16(5), pp. 299-311, 1993. https://doi.org/10.1002/jhrc.1240160507

[46] Juvancz, Z., Markides, K. E., Jicsinszky, L. "Enantiomer separation of disopyramide with capillary electrophoresis using various cyclodextrins", Electrophoresis, 18(6), pp. 1002-1006, 1997. https://doi.org/10.1002/elps.1150180623

[47] Schmitt, T., Engelhardt, H. "Optimization of enantiomeric separations in capillary electrophoresis by reversal of the migration order and using different derivatized cyclodextrins", Journal of Chromatography A, 697(1-2), pp. 561-570, 1995. https://doi.org/10.1016/0021-9673(94)00838-Z

[48] Chang, S. C., Reid III, G. L., Chen, S., Chang, C. D., Armstrong, D. W. "Evaluation of a new polar-organic high-performance liquid chromatographic mobile phase for cyclodextrin-bonded chiral stationary phases", TrAC Trends in Analytical Chemistry, 12(4), pp. 144-153, 1993.

https://doi.org/10.1016/0165-9936(93)87016-Q
[49] Mandoli, A., Schurig, V. "Separation of Enantiomers by Inclusion Gas Chromatography: On the Influence of Water in the Molecular Complexation of Methyl 2-Chloropropanoate Enantiomers on the Modified $\gamma$-Cyclodextrin Lipodex-E", Chirality, 28(2), pp. 124-131, 2016.

https://doi.org/10.1002/chir.22557

[50] Bicchi, C., Artuffo, G., D’Amato, A., Pellegrino, G., Galli, A., Galli. M. "GC separation of the enantiomers of $\gamma$ - and $\delta$-lactones on a mixture of 2,6-dimethyl-3-trifluoroacetyl- $\gamma$-cyclodextrin and OV-1701", Journal of High Resolution Chromatography, 14(10), pp. 701-704, 1991. https://doi.org/10.1002/jhrc.1240141016

[51] Iványi, R., Jicsinszky, L., Juvancz, Z. "Permethyl monoamino $\beta$-cyclodextrin a new chiral selective agent for capillary electrophoresis", Chromatographia, 53(3-4), pp. 166-172, 2000. https://doi.org/10.1007/BF02491565

[52] Juvancz, Z., Grolimund, K., Schurig, V. "Pharmaceutical applications of a bonded cyclodextrin stationary phase", Journal of Mirocolumn Separations, 5(5), pp. 459-468, 1993. https://doi.org/10.1002/mcs.1220050510

[53] König, W. A., Steinbach, E., Ernst, K. "Phosgene - a versatile reagent for enantiomer separation by capillary gas chromatography", Journal of Chromatography A, 301, pp. 129-135, 1984. https://doi.org/10.1016/S0021-9673(01)89183-4

[54] Armstrong, D. W., Nome, F., Spino, L. A., Golden, T. D. "Efficient detection and evaluation of cyclodextrin multiple complex formation", Journal of American Chemical Society, 108(7), pp. 1418-1421, 1986.

https://doi.org/10.1021/ja00267a010

[55] Krait, S., Salgado, A., Chankvetadze, B., Gago, F., Scriba, G. K. E. "Investigation of the complexation between cyclodextrins and medetomidine enantiomers by capillary electrophoresis, NMR spectroscopy and molecular modeling", Journal of Chromatography A, 1567, pp. 198-210, 2018. https://doi.org/10.1016/j.chroma.2018.06.010

[56] Michalska, K., Bocian, W., Bednarek, E., Pałys, B., CieleckaPiontek, J. "Enantioselective recognition of sutezolid by cyclodextrin modified non-aqueous capillary electrophoresis and explanation of complex formation by means of infrared spectroscopy, NMR and molecular modelling", Journal of Pharmaceutical and Biomedical Analysis, 169, pp. 49-59, 2019. https://doi.org/10.1016/j.jpba.2019.02.033

[57] Gogolashvili, A., Tatunashvili, E., Chankvetadze, L., Sohajda, T., Szeman, J., Salgado, A., Chankvetadze, B. "Separation of enilconazole enantiomers in capillary electrophoresis with cyclodextrin-type chiral selectors and investigation of structure of selector-selectand complexes by using nuclear magnetic resonance spectroscopy", Electrophoresis, 38(15), pp. 1851-1859, 2017. https://doi.org/10.1002/elps.201700078

[58] Salgado, A., Chankvetadze, B. "Applications of nuclear magnetic resonance spectroscopy for the understanding of enantiomer separation mechanisms in capillary electrophoresis", Journal of Chromatography A, 1467, pp. 95-144, 2016. https://doi.org/10.1016/j.chroma.2016.08.060 
[59] Schurig, V., Juvancz, Z., Nicholson, G. J., Schmalzing, D. "Separation of enantiomers on immobilized polysiloxane-anchored permethyl- $\beta$-cyclodextrin (CHIRASIL-DEX) by supercritical fluid chromatography", Journal of High Resolution Chromatography, 14(1), pp. 58-62, 1991. https://doi.org/10.1002/jhrc.1240140115

[60] Vega, E. D., Lomsadze, K., Chankvetadze, L., Salgado, A., Scriba, G. K. E., Calvo, E., López, J. A., Crego, A. L., Marina, M. L., Chankvetadze, B. "Separation of enantiomers of ephedrine by capillary electrophoresis using cyclodextrins as chiral selectors: Comparative CE, NMR and high-resolution MS studies", Electrophoresis, 32(19), pp. 2640-2647, 2011.

https://doi.org/10.1002/elps.201100015

[61] Gogolashvili, A., Chankvetadze, L., Takaishvili, N., Salgado, A., Chankvetadze B. "Separation of terbutaline enantiomers in capillary electrophoresis with neutral cyclodextrin-type chiral selectors and investigation of the structure of selector-selectand complexes using nuclear magnetic resonance spectroscopy", Electrophoresis, 41(12), pp. 1023-1030, 2020.

https://doi.org/10.1002/elps.202000010

[62] Bikádi, Z., Fodor, G., Hazai, I., Hári, P., Szemán, J., Szente, L., Fülöp, F., Péter, A., Hazai, E. "Molecular Modeling of Enantioseparation of Phenylazetidin Derivatives by Cyclodextrins", Chromatogtaphia, 71(S1), pp. 21-28, 2010. https://doi.org/10.1365/s10337-009-1461-9

[63] Felinger, A. "Data Analysis and Signal Processing in Chromatography", Elsevier, Amsterdam, Netherland, 1998.

[64] Chankvetadze, B., Schulte, G., Blaschke, G. "Reversal of enantiomer elution order in capillary electrophoresis using charged and neutral cyclodextrins", Journal of Chromatography A, 732(1), pp. 183-187, 1996.

https://doi.org/10.1016/0021-9673(95)01245-1

[65] Chankvetadze, B. "Enantiomer migration order in chiral capillary electrophoresis", Electrophoresis, 23(22-23), Special Issue: Capillary Electrophoresis and Electrochromatrography Reviews: Fundamentals, pp. 4022-4035, 2002. https://doi.org/10.1002/elps.200290016

[66] Juvancz, Z., Kiss, V., Schindler, J., Bálint, J. "Use of Achiral Derivatization to Increase Selectivity and Reverse the Order of Elution of Enantiomers on Chirasil-Dex", Chromatographia, 60(S1), pp. S161-S163, 2004. https://doi.org/10.1365/s10337-004-0292-y

[67] Armstrong, D. W., Li, W., Pitha, J. "Reversing enantioselectivity in capillary gas chromatography with polar and nonpolar cyclodextrin derivative phases", Analytical Chemistry, 62(2), pp. 214-217, 1990.

https://doi.org//10.1021/ac00201a023

[68] Salgado, A., Tatunashvili, E., Gogolashvili, A., Chankvetadze, B., Gago, F. "Structural rationale for the chiral separation and migration order reversal of clenpenterol enantiomers in capillary electrophoresis using two different $\beta$-cyclodextrins", Physical Chemistry Chemical Physics, 19(41), pp. 27935-27939, 2017.

https://doi.org/10.1039/c7cp04761d

[69] Juvancz, Z., Markides, K. E., Jicsinszky, L., Iványi, R. "Chiral selective separation of tocainide by capillary electrophoresis using various cyclodextrin derivatives", Journal of Microcolumn Separations, 13(2), pp. 62-68, 2001.

https://doi.org/10.1002/mcs.1022
[70] Konjaria M. L., Scriba, G. K. E. "Enantioseparation of analogs of the dipeptide alanyl-phenylalanine by capillary electrophoresis using neutral cyclodextrins as chiral selectors", Journal of Chromatography A, 1623, Article number: 461158, 2020. https://doi.org/10.1016/j.chroma.2020.461158

[71] Chankvetadze, B. "Recent trends in preparation, investigation and application of polysaccharide-based chiral stationary phases for separation of enantiomers in high-performance liquid chromatography", TrAC Trends in Analytical Chemistry, 122, Article number: 115709, 2020. https://doi.org/10.1016/j.trac.2019.115709

[72] Berthod, A., Chang, C.-D., Armstrong, D. W. " $\beta$-Cyclodextrin chiral stationary phases for liquid chromatography. Effect of the spacer arm on chiral recognition", Talanta, 40(9), pp. 1367-1373, 1993.

https://doi.org/10.1016/0039-9140(93)80212-A

[73] Schurig, V., Schmidt, R. "Extraordinary chiral discrimination in inclusion gas chromatography. Thermodynamics of enantioselectivity between a racemic perfluorodiether and a modified $\gamma$-cyclodextrin", Journal of Chromatography A, 1000(1-2), pp. 311-324, 2003.

https://doi.org/10.1016/S0021-9673(03)00180-8

[74] Jung, M., Schmalzing, D., Schurig, V. "Theoretical approach to the gas chromatographic separation of enantiomers on dissolved cyclodextrin derivatives", Journal of Chromatography A, 552, pp. 43-57, 1991.

https://doi.org/10.1016/S0021-9673(01)95922-9

[75] Levkin, P. A., Levkina, A., Czesla, H., Schurig, V. "TemperatureInduced Inversion of the Elution Order of Enantiomers in Gas Chromatography: N-Ethoxycarbonyl Propylamides and N-Trifluoroacetyl Ethyl Esters of $\alpha$-Amino Acids on ChirasilVal-C11 and Chirasil-Dex Stationary Phases", Analytical Chemistry, 79(12), pp. 4401-4409, 2007.

https://doi.org/10.1021/ac062064j

[76] Patil, R., Weatherly, C. A., Armstrong, D. W. "Chiral Gas Chromatography", In: Polavarapu, P. L. (ed.) Chiral Analysis, Elsevier, Amsterdam, Netherland, 2018, pp. 468-505. https://doi.org/10.1016/B978-0-444-64027-7.00012-4

[77] Cagliero, C., Sgorbini, B., Cordero, C., Liberto, E., Rubiolo, P., Bicchi, C. "Cyclodextrin Derivatives as Stationary Phases for the GC Separation of Enantiomers in the Flavor and Fragrance Field", In: Engel, K. H., Takeoka, G. (eds.) Importance of Chirality to Flavor Compounds, American Chemical Society, Washington, DC, USA, 2015, pp. 15-34.

https://doi.org/10.1021/bk-2015-1212.ch002

[78] Hardt, I., König, W. A. "Diluted versus undiluted cyclodextrin derivates in capillary gas chromatography and the effect of linear carrier gas velocity, column temperature, and length on enantiomer separation", Journal of Microcolumn Separations, 5(1), pp. 35-40, 1993.

https://doi.org/10.1002/mcs.1220050105

[79] Cagliero, C., Galli, S., Galli, M., Elmi, I., Belluce, M., Zampolli, S., Sgorbini, B., Rubiolo, P., Bicchi, C. "Conventional and enantioselective gas chromatography with microfabricated planar columns for analysis of real-world samples of plant volatile fraction", Journal of Chromatography A, 1429, pp. 329-339, 2016. https://doi.org/10.1016/j.chroma.2015.12.037 
[80] Schurig, V., Jung, M., Mayer, S., Fluck, M., Negura, S., Jakubetz, H. "Unified enantioselective capillary chromatography on a ChirasilDEX stationary phase. Advantages of column miniaturization", Journal of Chromatography A, 694(1), pp. 119-128, 1995. https://doi.org/10.1016/0021-9673(94)01075-p

[81] Armstrong, D. W., Tang, Y., Ward, T., Nichols, M. "Derivatized cyclodextrins immobilized on fused-silica capillaries for enantiomeric separations via capillary electrophoresis, gas chromatography, or supercritical fluid chromatography", Analytical Chemistry, 65(8), pp. 1114-1117, 1993.

https://oi.org/10.1021/ac00056a028

[82] Elbashir, A. A., Aboul-Enein, H. Y. "Multidimensional Gas Chromatography for Chiral Analysis", Critical Reviews in Analytical Chemistry, 48(5), pp. 416-427, 2018. https://doi.org/10.1080/10408347.2018.1444465

[83] Wrenn, S. "The Separation of Enantiomers by Capillary Electrophoresisa", Vieweg+Teubner Verlag, Wiesbaden, Germany, 2001.

[84] Lomsadze, K., Martínez-Girón, A. B., Castro-Puyana, M., Chankvetadze, L., Crego, A. L., Salgado, A., Marina, M. L., Chankvetadze, B. "About the role of enantioselective selector-selectand interactions and the mobilities of diastereomeric associates in enantiomer separations using CE", Electrophoresis, 30(16), pp. 2803-2811, 2009 https://doi.org/10.1002/elps.200900076

[85] Chankvetadze, B., Lindner, W., Scriba, G. K. E. "Enantiomer Separations in Capillary Electrophoresis in the Case of Equal Binding Constants of the Enantiomers with a Chiral Selector: Commentary on the Feasibility of the Concept", Analytical Chemistry, 76(14), pp. 4256-4260, 2004. https://doi.org/10.1021/ac0355202

[86] Beaufour, M., Morin, P., Ribet, J. P. "Chiral separation of the four stereoisomers of a novel antianginal agent using a dual cyclodextrin system in capillary electrophoresis", Journal of Separation Science, 28(6), pp. 529-533, 2005. https://doi.org/10.1002/jssc.200400097

[87] Papp, L. A., Hancu, G., Gyéresi, Á., Kelemen, H., Szabó, Z. I., Noszál, B., Dubský, P., Tóth, G. "Chiral separation of lansoprazole and rabeprazole by capillary electrophoresis using dual cyclodextrin systems", Electrophoresis, 40(21), pp. 2799-2805, 2019. https://doi.org/10.1002/elps.201900107
[88] Servais, A. C., Fillet, M. "Application of Dual-Cyclodextrin Systems in Capillary Electrophoresis Enantioseparations", In: Scriba, G. K. E. (ed.) Chiral Separations, Humana, New York, NY, USA, 2019, pp. 357-364. https://doi.org/10.1007/978-1-4939-9438-0_19

[89] Müllerová, L., Dubský, P., Gaš, B. "Twenty years of development of dual and multi-selector models in capillary electrophoresis: A review", Electrophoresis 35(19), pp. 2688-2700, 2014. https://doi.org/10.1002/elps.201400149

[90] Varga, E., Sohajda, T., Juvancz, Z., Bodane Kendrovics, R., Szekely, E., Bansaghi, G. "Development of Electrophoretic Methods for Simultaneous Determination of Enantiomeric Ratio and Composition of Diastereomeric Salt Mixtures", Chromatographia, 78(13-14), pp. 881-888, 2015. https://doi.org/10.1007//10337-015-2923-X

[91] Rudaz, S., Le Saux, T., Prat, J., Gareil, P., Veuthey, J. L. "Ultrashort partial-filling technique in capillary electrophoresis for infinite resolution of tramadol enantiomers and its metabolites with highly sulfated cyclodextrins", Electrophoresis 25(16), pp. 2761-2771, 2004.

https://doi.org/10.1002/elps.200406052

[92] Tanaka, Y. "Method development of enantiomer separations by affinity capillary electrophoresis, cyclodextrin electrokinetic chromatography and capillary electrophoresis-mass spectrometry", Se Pu, 20(4), pp. 317-327, 2002.

[93] Skelley, A. M., Mathies, R. A. "Chiral separation of fluorescamine-labeled amino acids using microfabricated capillary electrophoresis devices for extraterrestrial exploration", Journal of Chromatography A, 1021(1-2), pp. 191-199, 2003. https://doi.org/10.1016/j.chroma.2003.08.096

[94] Piehl, N., Ludwig, M., Belder, D. "Subsecond chiral separations on a microchip", Electrophoresis, 25(21-22), pp. 3848-3852, 2004. https://doi.org/10.1002/elps.200406028 